\title{
DESIGUALDADES DE OPORTUNIDADES EDUCACIONAIS NO INÍCIO DA TRAJETÓRIA ESCOLAR NO CONTEXTO BRASILEIRO1
}

\author{
Mariane Campelo Koslinski ${ }^{\mathrm{a}}$ \\ aUniversidade Federal do Rio de Janeiro (UFRJ). \\ Rio de Janeiro, RJ. Brasil. E-mail: mckoslinski@ufrj.br \\ Orcid: 0000-0002-9644-5041 \\ Tiago Lisboa Bartholo ${ }^{b}$ \\ ${ }^{b}$ Universidade Federal do Rio de Janeiro (UFRJ). \\ Rio de Janeiro, RJ, Brasil.E-mail: tiagobartholo@ufrj.br \\ Orcid: 0000-0002-2400-8707 \\ http://dx.doi.org/10.1590/0102-215245/110
}

\section{Introdução}

As desigualdades de oportunidades educacionais no contexto brasileiro são documentadas por inúmeros estudos que mostram o peso de marcadores sociais nas transições educacionais e a persistência de desigualdades que, após ampla expansão dos sistemas de ensino, são gradualmente transferidas para etapas subsequentes da escolarização (Hasenbalg e Silva, 2003; Mont'Alvão, 2011; Ribeiro, Ceneviva e Alves de Brito, 2015; Tavares Júnior, 2018). A disseminação dos sistemas de avaliação em larga escala, desde a década de 1990, permitiu a investigação de outras

\footnotetext{
1 O estudo longitudinal descrito neste artigo contou com o apoio das seguintes instituições: Banco Interamericano de Desenvolvimento, Fundação Maria Cecília Souto Vidigal, Fundação de Amparo à Pesquisa do Estado do Rio de Janeiro, Conselho Nacional de Desenvolvimento Científico e Tecnológico, e Instituto Alfa e Beto.
} 
nuances e facetas desse fenômeno, como a identificação do peso da origem socioeconômica da família e da composição do alunado sobre o desempenho e/ou a aprendizagem, bem como a identificação de fatores escolares capazes de promover maior equidade, ou seja, de atenuar estas desigualdades. Novamente, mesmo diante da expansão do acesso à educação e da sensível melhora no seu fluxo observada ao longo das últimas três décadas, as evidências apresentadas indicam não somente a persistência das desigualdades de oportunidades no que diz respeito à aprendizagem e ao desempenho, mas também o crescimento da distância entre alunos de diferentes origens socioeconômicas (Alves, Soares e Xavier, 2016; Soares e Delgado, 2016).

Recentemente, observamos no Brasil importantes movimentos em direção à expansão da educação compulsória que, a partir de 2009, passou a compreender a etapa da pré-escola. Tais movimentos foram acompanhados por 216 marcos legais que têm contribuído para a ampliação do acesso à educação infantil. Por exemplo, o Plano Nacional de Educação (PNE) previa a universalização do acesso de crianças de 4 a 5 anos à pré-escola até 2016 (Brasil, 2014). Ainda não alcançamos essa meta no Brasil, mas observamos a expansão da taxa de cobertura de 64,4\%, em 2001, para 93,8\% em 2018. Levando em conta diversas evidências produzidas por estudos com desenhos robustos (experimentais, quase experimentais e longitudinais) que indicam que a frequência à pré-escola traz efeitos em curto prazo para o desenvolvimento cognitivo e socioemocional das crianças, bem como, em mais longo prazo, para a aprendizagem e a trajetória escolar, em especial de crianças mais vulneráveis (Campbell et al., 2001; Peisner-Feinberg et al., 2001; Sammons et al., 2006; Schweinhart e Weikart, 1990; Sylva, Taggart e Siraj-Blatchford, 2003; Sylva et al., 2006; Sylva et al., 2010), a expansão da educação infantil, principalmente a virtual universalização da pré-escola, se apresenta 
como importante janela de oportunidade para diminuição das desigualdades educacionais no contexto brasileiro.

No entanto, contamos com escassos estudos com desenho longitudinal que permitam observar de forma adequada as desigualdades de oportunidades no início da escolarização no Brasil ou a partir de uma medida inicial sobre o desenvolvimento das crianças no início da pré-escola. Nesse contexto, o Laboratório de Pesquisa em Oportunidades Educacionais (LaPOpE), da Universidade Federal do Rio de Janeiro (UFRJ), desenvolveu um estudo longitudinal (Bartholo e Koslinski, 2020; Koslinski e Bartholo, 2019) com foco em crianças no início da pré-escola utilizando uma adaptação da ferramenta Performance Indicators in Primary Schools (Pips). A pesquisa iniciou a coleta de dados em 2017 e até o momento possui informações de dois municípios, com três amostras: duas nas redes públicas municipais e outra na rede privada e conveniada de uma das cidades. Os resultados elencados no presente artigo apresentam limitações quanto à validade externa. A amostra não é representativa do contexto brasileiro, e os resultados devem ser interpretados com cautela. Contudo, os dados coletados permitem desenvolver de análises que possibilitam a compreensão de padrões de desigualdades educacionais no início da escolarização obrigatória no Brasil, bem como estimar o efeito de programas focados na oferta da pré-escola e de características e processos escolares que incidem sobre tal fenômeno.

Este artigo, na sua primeira parte, discute brevemente a importância de estudos longitudinais, em especial com foco na pré-escola e/ou no início da escolarização obrigatória, para compreender padrões de desigualdades de oportunidades educacionais. Também descreve as características, potencialidades e fragilidades dos desenhos dos estudos em eficácia escolar com foco no início da trajetória de escolarização produzidos no contexto brasileiro. Em seguida, apresenta o desenho, as amostras e os instrumentos de coleta 
de dados utilizados no estudo longitudinal realizado pelo LaPOpE/UFRJ com crianças de 4 a 6 anos matriculadas na etapa da pré-escola. Desenvolvemos o estudo com três amostras, que compreenderam 123 escolas (6.379 crianças) públicas e privadas de dois municípios brasileiros. A terceira parte pontua algumas evidências trazidas pelas análises realizadas no âmbito deste estudo que nos permitem compreender desigualdades de oportunidades educacionais no início da escolarização obrigatória no contexto brasileiro e como o desenho do estudo permite identificar possíveis programas educacionais e práticas escolares capazes de atenuar distâncias no desenvolvimento de crianças de origens socioeconômicas distintas. Por fim, este artigo discute algumas limitações e fragilidades do estudo realizado, além de apontar lacunas a serem preenchidas e caminhos a serem percorridos por futuros trabalhos para ampliar a compreensão sobre o padrão de desigualdades no início da 218 pré-escola no contexto brasileiro, bem como para identificar os efeitos da expansão da pré-escola sobre esse fenômeno.

\section{Estudos longitudinais do início da trajetória escolar}

Diversos estudos realizados nos EUA e no Reino Unido indicam que frequentar a pré-escola é uma medida efetiva para garantir maior igualdade de oportunidades educacionais. Ou seja, contribui para o desenvolvimento de habilidades cognitivas e socioemocionais em curto e médio prazo, como também exerce impacto na trajetória escolar futura, e o seu efeito é mais acentuado para crianças vulneráveis oriundas de contextos socioeconômicos mais baixos (Barnett e Boocock, 1998; Campbell et al., 2001; NICHD Early Child Care Research Network, 2006; Peisner-Feinberg et al., 2001; Schweinhart e Wikart, 1990; Sylva et al., 2010).

Os benefícios observados ocorrem, em especial, para crianças que tiveram oportunidade de frequentar escolas de 
qualidade, mesmo que as medidas de qualidade da pré-escola, bem como os resultados observados (em curto, médio ou longo prazo), variem entre os estudos. Por exemplo, alguns trabalhos identificam relações consistentes entre a qualidade dos ambientes e processos em sala e o desenvolvimento cognitivo e/ou socioemocional durante a pré-escola, principalmente de crianças de nível socioeconômico mais baixo. ${ }^{2}$ Em alguns casos, esses efeitos ainda seriam observados em anos subsequentes, no início da escolarização primária. Entretanto, as evidências são menos consistentes para o impacto de características da estrutura da oferta da educação infantil - como formação dos professores, tempo do atendimento (parcial ou integral), razão adultos-crianças, características do prédio frequentado - sobre o desenvolvimento cognitivo das crianças durante a pré-escola (Howes et al., 2008; NICHD Early Child Care Research Network, 2006; Peisner-Feinberg et al., 2001; Sylva, Taggart e Siraj-Blatchford, 2003; Sylva et al., 2006; Sylva et al., 2010; Walston e West, 2004). Por fim, Sylva et al. (2006) também observaram o impacto de frequentar uma pré-escola de qualidade - medido a partir do valor agregado pelas escolas ao desenvolvimento cognitivo das crianças durante essa etapa no desempenho das crianças no início da escolarização primária. O estudo notou um impacto protetor em especial para crianças de nível socioeconômico mais baixo.

Na mesma direção, outro estudo, realizado por Tymms, Merrell e Henderson (2000) no Reino Unido, observou a

\footnotetext{
2 Nesses estudos, a qualidade dos ambientes foi mensurada a partir de instrumentos de observação de sala, como o Early Childhood Environment Rating Scale - Revised (Ecers-R), que inclui subescalas de espaço e mobiliário, rotinas de cuidado pessoal, linguagem-raciocínio, atividades de aprendizagem, interação, estrutura do programa e pais e funcionários (Harms, Clifford e Cryer, 2005); o Early Childhood Environment Rating Scale extension: four curricular subscales (Ecers-E), que inclui as subescalas de literacia, matemática, ciências e meio ambiente, e diversidade (Sylva, Taggart e Siraj-Blatchford, 2003); e o Classroom Assessment Scoring System (Class), que abrange dimensões como apoio emocional, organização da sala e apoio instrucional (Pianta, La Paro e Hamre, 2008).
} 
importância de frequentar uma escola de qualidade no primeiro ano de escolarização (reception year, série escolar que as crianças frequentam com 4 anos de idade) para o desenvolvimento cognitivo das crianças três anos depois, no $3^{\circ}$ ano da escolarização primária. De forma similar à pesquisa de Sylva et al. (2006), esse estudo mediu a qualidade da oferta no primeiro ano de escolarização a partir do valor agregado pelas escolas em linguagem, vocabulário e matemática. Em trabalho posterior, com duração de 7 anos, Tymms et al. (2009) observaram que o progresso relativo de cada ano na trajetória escolar tinha impacto no resultado do $6^{\circ}$ ano da educação primária (quando os alunos têm 11 anos). No entanto, notou-se que frequentar uma escola com alta qualidade no primeiro ano de escolarização (reception class) exercia maior efeito em longo prazo (Tymms et al., 2009). As evidências encontradas fortalecem a ideia de que o início da escolarização obrigatória representa uma janela de oportunidades no desenvolvi220 mento, uma vez que seus efeitos são mais fortemente sentidos em longo prazo. Portanto, podemos esperar que a expansão da oferta de um ensino de qualidade no início da trajetória escolar possa exercer um impacto mais acentuado na diminuição das desigualdades educacionais.

Os estudos citados têm em comum desenhos robustos para estimar os efeitos observados, como desenhos experimentais e, mais frequentemente, longitudinais. O desenho de pesquisa longitudinal, de acordo com Goldstein (1997), é um dos itens-chave que permitem estimar com maior precisão a relação entre os fatores e os resultados/desfechos escolares. ${ }^{3}$ De acordo com o autor, na ausência ou impossibilidade de realizar estudos experimentais para realizar "comparações justas"

\footnotetext{
3 Outras condições para identificação de fatores escolares associados ao desempenho dos alunos mencionadas pelo autor incluem: o uso de modelos de regressão multinível, que permitem investigar a "eficácia diferencial" das escolas e/ou professores; a replicação das análises no tempo e no espaço; e que o pesquisador apresente uma teoria plausível para explicar os resultados obtidos (Goldstein, 1997).
} 
entre as escolas e/ou evitar viés de seleção, seria necessário um controle por meio de características que sabemos ter impacto sobre os resultados escolares de interesse a partir das teorias disponíveis (Goldstein, 1997). Por exemplo, já contamos com uma vasta gama de estudos que mostram que a composição do alunado (características relacionadas a sexo, origem socioeconômica e grupos étnicos) e sua habilidade/desempenho inicial diferem entre escolas por causa de diversos fatores e, portanto, para evitar viés de seleção, seria necessário medir tais dimensões de forma acurada no âmbito individual (Fitz-Gibbon, 1996; Goldstein, 1997; Lee, 2004). Fitz-Gibbon (1996), ao descrever pré-requisitos de desenhos adequados para sistemas de monitoramento na educação, argumenta que os agrupamentos nas escolas são resultado de vários processos de autosseleção e, por isso, podemos esperar que os grupos se diferenciem de diversas formas que não conseguimos mensurar. Assim, medidas de habilidade/ desempenho iniciais permitem realizar o controle de forma mais efetiva, ou comparações mais justas, se comparados com desenhos transversais que compreendem somente controles contextuais dos alunos. Por esse motivo, é importante que a pesquisa seja longitudinal, com duas ou mais medidas sobre os mesmos indivíduos ao longo do tempo, permitindo o controle, de forma adequada, das diferenças preexistentes entre os alunos (Fitz-Gibbon, 1996; Goldstein, 1997).

No Brasil, com a expansão dos sistemas de avaliação externa da educação, observamos a proliferação de estudos com foco na primeira etapa do ensino fundamental que buscaram compreender padrões e tendências das desigualdades de oportunidades educacionais e identificar fatores escolares associados à proficiência dos alunos (Alves e Xavier, 2016; Alves, Soares e Xavier, 2016; Bonamino et al., 2010; Franco et al., 2007; Soares, 2004; Soares, 2003; Soares e Delgado; 2016). Tais estudos assinalaram a associação de fatores escolares - como infraestrutura das escolas, 
clima escolar/acadêmico, perfil da liderança/gestão, ênfase e métodos pedagógicos, entre outros - e extraescolares com o desempenho dos alunos, seja no $5^{\circ}$ ou no $3^{\circ}$ ano do ensino fundamental. Também buscaram identificar fatores escolares associados à equidade, isto é, que contribuíam para diminuir as desigualdades de desempenho entre alunos de origens distintas no que diz respeito a nível socioeconômico, cor/raça e/ou sexo. No entanto, esses estudos, em sua maioria, utilizaram dados seccionais que apresentavam limitações para estimar fatores escolares associados ao desempenho dos alunos. Além do viés de seleção mencionado, os dados seccionais se referem a um agregado de aprendizado ao longo do tempo. Já as medidas de condições escolares (como aquelas das avaliações externas) se referem ao ano em que o dado foi coletado, de modo que faltaria uma sincronia temporal entre elas (Franco, Brooke e Alves, 2008). Citamos os estudos de Castro (2017), Marino (2016) e 222 Bartholo e Costa $(2016)^{4}$ como exemplos dos raros estudos com desenho longitudinal com foco no início do ensino fundamental realizados no contexto brasileiro utilizando bases de dados das avaliações externas. Entretanto nenhum deles dispunha de uma medida de linha de base, ou seja, da entrada na escolarização obrigatória.

O único estudo longitudinal de grande escala realizado no contexto brasileiro com foco no início da escolarização obrigatória ${ }^{5}$ que contou com uma linha de base foi o Estudo Longitudinal da Geração Escolar 2005 (Geres 2005). Essa pesquisa seguiu uma coorte matriculada na $1^{\underline{a}}$ série (equivalente ao atual $2^{\circ}$ ano) do ensino fundamental, a partir do primeiro semestre de 2005, durante quatro anos. Foram realizadas cinco coletas de dados com os mesmos alunos,

\footnotetext{
4 Os três estudos mencionados utilizaram dados dos sistemas de avaliação municipal da cidade do Rio de Janeiro, quais sejam, o Alfabetiza Rio e/ou a Prova Rio.

5 No momento em que o estudo foi realizado, o ensino fundamental de oito anos era o período de escolarização obrigatória no Brasil para a faixa etária de 7 a 14 anos.
} 
em uma amostra de mais de 300 escolas públicas e privadas (somando aproximadamente 21.550 alunos) localizadas em cinco cidades: Rio de Janeiro, Belo Horizonte, Campinas, Campo Grande e Salvador (Brooke e Bonamino, 2011).

A pesquisa tinha como objetivo principal identificar as características escolares que: (1) maximizam a aprendizagem dos alunos e minimizam o impacto da origem social sobre o aprendizado; (2) diminuem a probabilidade de repetência dos alunos; e (3) reduzem a probabilidade de absenteísmo (Franco, Brooke e Alves, 2008). Esse estudo teve início há quinze anos e trouxe importante contribuição para compreender padrões de desigualdades no início da escolarização obrigatória, com a observação de diferentes patamares no ponto de partida e de padrões das curvas de crescimento da proficiência dos alunos em língua portuguesa e matemática (Brooke e Bonamino, 2011).

No entanto, desde a realização do Geres 2005, observamos a expansão do ensino fundamental, que passou a ter nove anos e a ser obrigatório para crianças de 6 a 14 anos em 2006. ${ }^{6}$ Em seguida, em 2009, a pré-escola passou a ser etapa da escolarização obrigatória, com previsão de universalização do atendimento a crianças de 4 e 5 anos até 2016. ${ }^{7}$ Não obstante, essa expansão não foi acompanhada por maior interesse ou foco das pesquisas, em especial no campo da sociologia da educação, sobre desigualdades escolares na educação infantil (Rodrigues, 2018). Também não localizamos, após a realização do Geres 2005, outros estudos longitudinais em larga escala com foco no período inicial da escolarização obrigatória.

\footnotetext{
6 A Lei n⿳o 11.274, de 6 de fevereiro de 2006, estabelece a duração de nove anos para o ensino fundamental, com matrícula obrigatória para crianças a partir dos 6 anos de idade.

7 A Emenda Constitucional no 59, de 11 de novembro de 2009, prevê a obrigatoriedade do ensino a partir dos 4 anos de idade, e a primeira meta do PNE (Lei no 13.005 , de 25 de junho de 2014) prevê a universalização do acesso à pré-escola.
} 
Desta forma, o Brasil ainda conta com poucos trabalhos relativos ao impacto da educação e da pré-escola na aprendizagem e trajetória escolar dos alunos que nos ajudem a compreender os possíveis efeitos da expansão da educação infantil e da universalização da pré-escola sobre as desigualdades educacionais (com especial foco nas desigualdades de aprendizagem) no contexto brasileiro. Citamos como um dos esforços nessa direção o estudo de Damiani et al. (2011), que observou que crianças que frequentam a pré-escola tendem a ter trajetórias escolares mais longas, com maior probabilidade de terminar o ensino fundamental e a educação básica. Esse estudo enfoca o efeito da frequência à creche e à pré-escola sobre a trajetória escolar, e não sobre o desenvolvimento ou desempenho escolar futuro das crianças. Além disso, apesar de contar com controles relacionados à origem socioeconômica, não apresenta controles em relação ao desenvolvimento das crianças ou à qualidade dos 224 estabelecimentos escolares frequentados e, portanto, apresenta limitações para estabelecer uma relação causal entre a frequência à pré-escola e os desfechos observados.

Já o estudo de Campos et al. (2011a) foi pioneiro na investigação da relação entre a qualidade dos ambientes da pré-escola (medida pela escala Ecers-R) e o resultado da aprendizagem de língua portuguesa de crianças no $2^{\circ}$ ano do ensino fundamental (medido pela Provinha Brasil). A pesquisa foi desenvolvida em escolas de três capitais brasileiras, e as análises indicaram que crianças que frequentaram instituições com boa qualidade na pré-escola apresentaram melhor desempenho no teste do que crianças que não frequentaram a pré-escola ou que o fizeram em instituições com níveis insatisfatórios de qualidade (Campos et al., 2011a). No entanto, o estudo descrito não apresentava um desenho longitudinal, mas utilizou um modelo hierárquico cruzado para estimar o desempenho das crianças na Provinha Brasil, com controles relacionados às características das famílias e das 
escolas frequentadas no ensino fundamental. As análises não contavam, porém, com controle do desenvolvimento prévio das crianças e, portanto, apresentavam limitações para lidar com o viés de seleção. Estudo mais abrangente realizado pelo mesmo grupo sobre a qualidade dos ambientes da pré-escola em seis municípios observou que precisaríamos avançar para alcançar certo padrão de qualidade na oferta dessa etapa de ensino (Campos et al., 2011b). Os resultados dos dois estudos nos trazem alguns indícios da necessidade de melhorar a qualidade dessa oferta para que a expansão da pré-escola exerça impacto positivo nas trajetórias escolares futuras das crianças e na desejada diminuição das desigualdades.

Por fim, o estudo de Silva et al. (2019), realizado com escolas da rede municipal de Boa Vista, investigou a associação entre indicadores de qualidade de processo e de desenvolvimento infantil durante a pré-escola utilizando uma adaptação do instrumento Measuring Early Learning Quality and Outcomes (MELQO). Os autores observaram uma relação positiva entre um indicador global de processos em sala (que incluía dimensões de qualidade das interações personalizadas e atividades não receptivas na turma) e o desenvolvimento das crianças nos domínios de linguagem e alfabetização, matemática, função executiva e desenvolvimento socioemocional. $\mathrm{O}$ estudo traz importante contribuição para o debate sobre a definição da qualidade da oferta da pré-escola e seus possíveis efeitos no desenvolvimento cognitivo das crianças em curto prazo, no início da escolarização obrigatória. Contudo, apresenta um desenho transversal que, novamente, traz limitações para a realização de inferências causais quanto ao efeito das características das escolas mensuradas sobre o desenvolvimento das crianças durante a pré-escola.

Assim, o trabalho em foco neste artigo surgiu da necessidade de construir desenhos de pesquisa mais robustos, que permitam maior qualidade na inferência causal para entender os efeitos específicos de processos escolares no início 
da escolarização obrigatória no Brasil. Apresentamos objetivos e características semelhantes às do Geres 2005, como um desenho longitudinal com linha de base no início da escolarização obrigatória. No entanto, o presente estudo adotou uma medida de base mais precoce, no momento inicial de ingresso na pré-escola (aos 4 anos de idade), em face da expansão da escolarização obrigatória no Brasil. Além disso, observa o desenvolvimento das crianças nessa etapa de forma mais abrangente, incluindo medidas sobre o desenvolvimento cognitivo, socioemocional e físico/motor das crianças. Por fim, utiliza os Pips como principal instrumento para acompanhar o desenvolvimento das crianças, um teste cognitivo adaptativo que tem aplicação individual e não orquestrada. Os Pips já foram adaptados e utilizados em diversos países, o que permite desenvolver comparações internacionais em relação aos fenômenos observados. As características do desenho e das amostras, bem como os 226 instrumentos utilizados, são detalhadas na seção a seguir.

\section{Desenho do estudo, amostras e instrumentos utilizados}

Entre os anos de 2017 e 2019, pesquisadores do LaPOpE/UFRJ analisaram o desenvolvimento de crianças em seus dois primeiros anos na escola (escolarização obrigatória). O estudo incluiu três amostras de crianças de 4 a 6 anos matriculadas na etapa da pré-escola em instituições públicas e privadas de duas cidades brasileiras.

Essa pesquisa foi realizada com o objetivo de compreender o desenvolvimento das crianças e padrões de desigualdades no início da escolarização obrigatória e identificar o impacto de políticas e programas escolares, bem como características da escola (liderança, clima escolar, enturmação, entre outros) e processos em sala (padrões de interação entre crianças e adultos e práticas pedagógicas) associados ao desenvolvimento das crianças durante a pré-escola. Para tanto, foram aplicados três instrumentos a fim de mensurar 
o desenvolvimento das crianças: (1) em relação à dimensão cognitiva, utilizou-se uma adaptação dos $\operatorname{Pips}^{8}$ ao contexto brasileiro como ferramenta para medir o desenvolvimento da linguagem e da matemática (Bartholo et al., 2020a; Bartholo et al., 2020b; Tymms, Merrell e Henderson, 1997; Tymms, Merrell e Jones, 2004); (2) aptidão física e coordenação motora foram avaliadas com o Teste Sentar e Levantar (TSL) (Aguiar, 2018; Aguiar e Bartholo, 2019; Aguiar, Bartholo e Tavares Júnior, 2019; ARAÚJO, 1999); e (3) comportamento e desenvolvimento pessoal, social e emocional (DPSE) foram aferidos com ferramenta desenvolvida pela Universidade de Durham e adaptada ao contexto brasileiro por pesquisadores da UFRJ (Merrell e Beiley, 2008; Santos, 2020).

Além do instrumento cognitivo dos Pips, do TSL e dos testes de comportamento e DPSE, este estudo também coletou dados contextuais dos alunos (características sociodemográficas, do ambiente de aprendizagem em casa, de pré-natal e prematuridade) e das escolas (incluindo políticas da instituição, perfil da liderança, clima escolar, experiência e formação inicial e continuada, expectativas, referências e práticas dos professores) a partir de questionários aplicados a

8 Os Pips são uma ferramenta acurada e testada por vinte anos no Reino Unido e em outros países, sendo composto pelas seguintes dimensões: (1) escrita; (2) vocabulário; (3) ideias sobre leitura - avalia conceitos sobre diferentes impressos; (4) consciência fonológica; (5) identificação de letras; (6) reconhecimento de palavras e leitura; (7) ideias sobre matemática; (8) contagem e números; (9) adição e subtração sem símbolos; (10) identificação de formas; e (11) identificação de números. Para mais informações sobre sua adaptação e aplicação no contexto brasileiro, ver Bartholo et al. (2020a; 2020b). A aplicação do teste é individual, com duração que varia entre 10 e 20 minutos. Os pesquisadores coletaram os dados com o auxílio de um tablet e de um programa que apresentava as questões e, dependendo do tipo de pergunta, a criança respondia apontando a resposta em um caderno ou oralmente. O pesquisador registrava a resposta na tela do tablet, e o programa selecionava a próxima pergunta. O programa é ajustado de forma que erros e acertos são considerados para continuar o procedimento, no caso de acertos consecutivos, ou interrompê-lo, quando a criança demonstra desconhecer determinado conteúdo. Cada sessão do teste apresenta itens com dificuldade crescente, de modo que ele tenha duração mínima, sem deixar a criança entediada com perguntas muito simples ou muito difíceis (Tymms, Merrell e Jones, 2004). 
pais/responsáveis, professores e diretores. Por fim, na amostra 1, além dos instrumentos descritos, também aplicamos a uma subamostra de turmas o Classroom Assessment Scoring System para a pré-escola (Class Pre-K) (Pianta, La Paro e Hamre, 2008). O Class Pre-K é um instrumento amplamente utilizado em pesquisas com foco na pré-escola que visa captar a qualidade de processos em sala com base em três dimensões: apoio emocional, organização da sala e apoio instrucional.

O estudo foi composto por três diferentes amostras, para as quais foram utilizados distintos desenhos de pesquisa e critérios de seleção de escolas, turmas e crianças. O Quadro 1 apresenta um resumo das amostras e dos dados coletados em cada uma delas.

Quadro 1

Descrição das amostras

\begin{tabular}{|l|c|c|c|c|l|}
\hline \multicolumn{1}{|c|}{ Amostras } & Amostra & $\begin{array}{c}\text { No de } \\
\text { escolas }\end{array}$ & $\begin{array}{c}\text { No de } \\
\text { turmas }\end{array}$ & $\begin{array}{c}\text { № de } \\
\text { crianças }\end{array}$ & \multicolumn{1}{|c|}{ Dados coletados* } \\
\hline $\begin{array}{l}\text { Amostra } \\
\text { 1: escolas } \\
\text { públicas da } \\
\text { cidade A }\end{array}$ & Probabilística & 46 & 123 & 4.302 & $\begin{array}{l}\text { O1: Pips e TSL. } \\
\text { O2: Pips, TSL, DPSE, } \\
\text { questionários contextuais } \\
\text { e para a escola. } \\
\text { O3: Pips, TSL, DPSE, Class, } \\
\text { questionários contextuais } \\
\text { e para a escola. }\end{array}$ \\
\hline $\begin{array}{l}\text { Amostra } \\
\text { 2: escolas } \\
\text { privadas da } \\
\text { cidade A }\end{array}$ & probabilística & 36 & 124 & 1.407 & $\begin{array}{l}\text { O1: Pips, TSL e } \\
\text { questionários contextuais. } \\
\text { O2: Pips, TSL, DPSE e } \\
\text { questionário para } \\
\text { a escola. }\end{array}$ \\
\hline $\begin{array}{l}\text { Amostra } \\
\text { 3: escolas } \\
\text { públicas da } \\
\text { cidade B }\end{array}$ & Probabilística & 41 & 120 & 670 & $\begin{array}{l}\text { 01: Pips, TSL e } \\
\text { questionários } \\
\text { contextuais. } \\
\text { O2: Pips, TSL, DPSE e } \\
\text { questionário para } \\
\text { a escola. }\end{array}$ \\
\hline Total & 123 & 367 & 6.379 & \\
\hline
\end{tabular}

Fonte: Elaboração própria.

*O1, O2 e O3 indicam a primeira, a segunda e a terceira onda de coleta de dados, respectivamente. 
Todas as amostras apresentam desenhos longitudinais, com ao menos duas medidas sobre o desenvolvimento das mesmas crianças. Há, no entanto, uma diferença importante entre elas. As amostras 2 e 3 tiveram dados coletados no início e no final do ano letivo de duas coortes de crianças matriculadas no primeiro e segundo ano da pré-escola. A amostra 1 acompanhou uma única coorte de crianças ao longo da pré-escola, com um total de três medidas sobre seu desenvolvimento das crianças ao longo do tempo. No primeiro ano da pré-escola foram coletados dados no início e no final do período letivo, e no segundo ano da pré-escola foi feita uma última coleta de dados ao final do ano letivo. Ambos os desenhos permitem estimar modelos de valor agregado, isto é, utilizar uma medida inicial (linha de base) como controle ou preditor da medida no final do ano.

Mesmo com as diferenças dos desenhos, o estudo feito com as três amostras nos permitiu obter uma medida inicial sobre o desenvolvimento das crianças no início da escolarização obrigatória. Como dito anteriormente, grande parte das avaliações externas, como a Avaliação Nacional do Rendimento Escolar (Anresc) - conhecida como Prova Brasil - e a Avaliação Nacional da Educação Básica (Aneb), coleta dados transversais, que trazem uma fotografia do final de etapas da escolarização. Mais recentemente, observamos outros sistemas de avaliação que permitem um retrato mais precoce, como a Avaliação Nacional de Alfabetização (ANA), aplicada no $3^{\circ}$ ano do ensino fundamental; mas, ainda assim, essas medições são feitas após um período de exposição à escolarização obrigatória.

Nesse sentido, os estudos sobre o efeito da escola no desempenho dos alunos no contexto brasileiro realizados com dados de avaliação externa, em sua maioria, apresentam desenhos transversais. Há riscos importantes que devem ser considerados quando analisamos resultados de 
estudos desse tipo. Os coeficientes obtidos podem superestimar os efeitos da escola e do professor. A omissão da principal variável de controle, a medida inicial sobre o desenvolvimento das crianças, aumenta o risco de inflar o coeficiente das demais variáveis presentes no modelo. Além disso, sem uma linha de base, a variável do nível socioeconômico não permite observar a desvantagem na aprendizagem em dado ano ou etapa escolar, uma vez que mensura o acúmulo da desvantagem pré-existente antes da entrada na escola e ao longo de toda a trajetória escolar. Nesse caso, é possível que o nível socioeconômico e outras variáveis sobre características da criança e de sua família apresentem resultados enganosos ou que confundam o pesquisador/leitor. Como mencionado anteriormente, um número importante de publicações baseadas em dados transversais sobre o efeito da escola no Brasil não satisfaz nem mesmo um dos critérios para estudos sobre eficácia 230 escolar destacados por Goldstein (1997) e corroborados pelos autores deste texto.

\section{0 que já sabemos sobre desigualdades de oportunidades educacionais no início da escolarização obrigatória}

Desde o início do estudo, pesquisadores e alunos de graduação e pós-graduação vinculados ao LaPOpE/ UFRJ produziram diversos trabalhos sobre fatores escolares e extraescolares associados ao desenvolvimento cognitivo das crianças durante os primeiros anos de escolarização obrigatória (Aguiar, 2018; Aguiar e Bartholo, 2019; Aguiar, Bartholo e Tavares Júnior, 2019; Bartholo e Koslinski, 2020; Bartholo et al., 2020a; Bartholo et al., 2020b; Koslinski e Bartholo, 2019; Mendonça, 2019; Oliveira, 2020; Santos, 2020; Siqueira, 2019).

Neste artigo, vamos relatar o que o estudo realizado com as amostras probabilísticas (1 e 3) nos permitiu, até o momento, compreender sobre os padrões de desigualdades 
no desenvolvimento das crianças no início e ao longo da pré-escola. Pretendemos ilustrar como os dados com características longitudinais coletados no estudo permitem estimar com maior precisão o efeito de programas e políticas educacionais no desenvolvimento das crianças e na promoção de equidade ao longo dos dois primeiros anos de escolarização obrigatória. Selecionamos três variáveis principais para debater neste texto. A primeira está relacionada com características da família, e outras duas com características escolares - infraestrutura e programas/escolas dedicadas à educação infantil.

A relação entre origem socioeconômica e desempenho/trajetória escolar do estudante é amplamente documentada na literatura educacional (Bonamino et al., 2010; Brooke e Bonamino, 2011; Coleman et al., 1966; Franco et al., 2007; Sirin, 2005; Soares, 2003; Soares, 2004). Pesquisas realizadas em sistemas educacionais de diversos países reforçaram esse achado, variando apenas a intensidade da associação entre as duas dimensões (Crahay e Baye, 2013). Em linhas gerais, uma maior associação entre o nível socioeconômico e o desempenho escolar pode ser interpretada como um indicativo de que o sistema escolar não promove equidade. Em outras palavras, os resultados e as trajetórias escolares dos estudantes são explicados por sua origem socioeconômica. Por outro lado, quando os resultados sugerem uma associação fraca ou negativa entre as duas dimensões supracitadas, o resultado pode ser tomado como um indicativo de diminuição das desigualdades educacionais e de promoção da equidade.

No estudo longitudinal, construímos um indicador de nível socioeconômico (NSE) com os dados coletados nos questionários respondidos pelos responsáveis das crianças. Para sua composição, utilizamos informações sobre a posse de determinados bens, características do domicílio e nível de escolaridade. Desse modo, os itens incluídos no indicador 
de NSE foram: (1) escolaridade dos pais; ${ }^{9}$ (2) participação em programa de transferência de renda; (3) densidade domiciliar; ${ }^{10}$ (4) quarto só para a criança; (5) acesso à internet; (6) carro; (7) TV por assinatura; (8) máquina de lavar; (9) computador; (10) tablet; e (11) impressora. A partir dos elementos descritos, o indicador de $\mathrm{NSE}^{11}$ foi elaborado utilizando o modelo de Rasch (Boone, 2016), através do software Winsteps. Esse procedimento possibilitou a análise teórica e empírica de cada item que compõe o indicador.

As análises apresentadas estimam a associação entre o indicador de NSE ao ingressar na pré-escola, no primeiro e no segundo ano na escola. Os dados foram analisados separadamente para as amostras probabilísticas das redes públicas de dois municípios brasileiros. Cabe ressaltar que as medidas do teste cognitivo e o indicador de NSE foram calculados de forma idêntica, o que ajuda na comparação dos coeficientes. A Tabela 1 apresenta, nas primeiras colunas, os coeficientes do 232 indicador de NSE estimados por modelos de regressão linear ${ }^{12}$ para o ponto de partida do desenvolvimento das crianças (ao entrar na pré-escola), em linguagem e matemática. Nas colunas seguintes, apresentam-se os coeficientes do indicador de NSE estimados a partir de modelos de regressão multinível, ${ }^{13}$

9 A escolaridade dos pais foi recodificada da seguinte maneira: (1) para pais com alta escolaridade e (0) para pais de baixa escolaridade. Foram considerados de alta escolaridade pais que concluíram o ensino médio ou grau superior. Pais de baixa escolaridade são aqueles que não concluíram o ensino médio ou com grau inferior de escolaridade.

${ }^{10} \mathrm{O}$ indicador de densidade domiciliar foi calculado pela razão entre o número de pessoas e o número de quartos disponíveis. Em seguida, essa razão foi recodificada em uma variável dicotômica que indica que o domicílio tem adensamento igual ou superior a três indivíduos por dormitório.

11 O indicador de NSE foi calculado somente para os alunos cujos pais/responsáveis tivessem respondido sua escolaridade ou no mínimo dois itens.

12 No modelo foram incluídas como covariáveis a idade da criança, medida em meses de vida, e o sexo.

13 Quanto aos modelos multinível, foram incluídas no nível 1 (criança) variáveis de controle referentes a idade, sexo e medidas de desenvolvimento cognitivo anteriores, e no nível 2 (escola) o NSE agregado pelas escolas. 
com medidas de valor agregado. Nesses modelos os coeficientes expressam a associação do NSE das crianças com a aprendizagem de linguagem e matemática em cada ano da pré-escola. Todos os coeficientes são reportados em effect size - para maiores detalhes, ver Tymms (2004). Um número crescente de estudos no campo da educação tem optado por reportar seus resultados em effect size, e há extenso debate sobre as melhores formas de interpretá-los (Higgins et al., 2013).

Tabela 1

Relação entre o NSE e o desenvolvimento cognitivo no ingresso e ao longo da pré-escola

\begin{tabular}{|c|c|c|c|c|c|c|c|}
\hline \multicolumn{2}{|c|}{} & \multicolumn{2}{|c|}{ Ponto de partida } & \multicolumn{2}{c|}{1 10 ano da pré-escola } & \multicolumn{2}{c|}{ 20 ano da pré-escola } \\
\hline \multicolumn{2}{|c|}{} & Linguagem & Matemática & Linguagem & Matemática & Linguagem & Matemática \\
\hline \multirow{2}{*}{$\begin{array}{c}\text { Amostra } \\
\text { da rede } \\
\text { A }\end{array}$} & $\begin{array}{c}\text { Effect } \\
\text { size }\end{array}$ & 0,341 & 0,237 & 0,094 & 0,103 & $-0,149$ & $-0,082$ \\
\cline { 2 - 8 } & p-valor & 0,002 & 0,029 & 0,428 & 0,385 & 0,242 & 0,510 \\
\hline $\begin{array}{c}\text { Amostra } \\
\text { da } \\
\text { rede B }\end{array}$ & $\begin{array}{c}\text { Effect } \\
\text { size }\end{array}$ & 0,485 & 0,515 & 0,195 & 0,258 & 0,149 & 0,076 \\
\cline { 2 - 9 } & p-valor & 0,000 & 0,000 & 0,000 & 0,000 & 0,001 & 0,094 \\
\hline
\end{tabular}

Fonte: Elaboração própria.

Os resultados apresentam alguns padrões que permitem interpretações preliminares sobre a relação entre o nível socioeconômico e o nível de desenvolvimento ao ingressar na pré-escola. Talvez mais importantes, os resultados dos modelos de valor agregado (desenvolvimento ao longo da pré-escola) possibilitam observar se há alguma indicação de aumento ou diminuição das desigualdades educacionais.

Em ambas as redes de ensino se observa uma correlação positiva e estatisticamente significativa para o ponto de partida em linguagem e matemática. Esse resultado é esperado, em grande medida, e corrobora o que outras pesquisas no Brasil reportam a respeito das demais etapas do ensino. A força da correlação entre o desenvolvimento no ponto de 
partida e o NSE é diferente quando comparamos as duas redes de ensino, e há várias hipóteses que não são autoexcludentes e podem explicar esse resultado. Por exemplo, diferenças na composição social dos alunos matriculados na rede pública, diferenças importantes das matrículas nas redes pública e privada, políticas intersetoriais que impactam o desenvolvimento infantil ou, ainda, cobertura da creche (de crianças entre 0 e 3 anos). O estudo longitudinal não é capaz de testar nenhuma dessas hipóteses.

Os coeficientes dos modelos de valor agregado para o primeiro e o segundo ano na escola também apresentam um padrão. Nenhum dos coeficientes estimados para a rede de ensino A é estatisticamente significativo, e a direção da associação não é clara. Um padrão diferente pode ser observado na rede de ensino $\mathrm{B}$, para a qual todos os coeficientes são estatisticamente significativos com $\mathrm{p} \leq 0,01$, com exceção do coeficiente para matemática no segundo ano na escola. 234 Os dados sugerem que, mesmo com o controle por ponto de partida (medida inicial do desenvolvimento), sexo e idade das crianças, alunos com maior nível socioeconômico aprendem mais. Esse resultado deve ser interpretado com cautela, mas sugere que o sistema pode ampliar as desigualdades educacionais nos primeiros dois anos da escolarização obrigatória.

Há aqui algumas reflexões importantes a serem feitas. A primeira é que, quando pensamos em políticas educacionais, em especial aquelas focadas na primeira infância e no ensino fundamental, é importante olhar individualmente para cada município. A formulação e implementação de programas pode apresentar grande variação, o que tem potencial para explicar certos resultados. O segundo ponto reforça mais uma vez que a política educacional importa e parece explicar pelo menos parte da variação observada na desigualdade educacional.

Os modelos de valor agregado são muito importantes para avaliarmos o fenômeno do aprendizado e sua relação 
com as redes de ensino. Os mesmos coeficientes, se apresentados apenas com modelos transversais, contariam uma história diferente. $\mathrm{O}$ resultado de ambas as redes seria muito parecido, ou seja, ao associar o NSE com o desenvolvimento cognitivo no início e no final da pré-escola, se apresentariam diferenças somente no que diz respeito à intensidade da associação nas duas redes. Apenas com a introdução do ponto de partida no modelo de valor agregado é que esse cenário muda. Nesse caso, passamos a identificar que redes públicas no Brasil podem apresentar resultados bastante distintos quando pensamos nas oportunidades educacionais e na equidade do sistema.

A segunda variável de interesse é uma característica relacionada à infraestrutura das escolas: diversos estudos já estimaram o efeito do tamanho da turma no aprendizado dos alunos (Finn e Achilles, 1999; Perlman et al., 2017). Afinal, faz diferença para o estudante ser alocado em turmas menores, ou seja, com menos estudantes?

Pesquisas internacionais sobre o efeito do tamanho da turma apresentam resultados mistos, pois algumas sugerem que turmas menores potencializam o aprendizado, e outras indicam não haver efeito sobre este (Finn e Achilles, 1999; Perlman et al., 2017). No entanto, esses resultados devem ser interpretados com cuidado, principalmente quando refletimos sobre o contexto das escolas no Brasil. Boa parte dos estudos que apresentam dados com características longitudinais e modelo hierárquico sobre o efeito do tamanho das turmas foi realizada nos EUA ou em países europeus. É incomum nesses países encontrar turmas, para a faixa etária de 4 a 6 anos, com tamanho superior a 15 alunos. Por exemplo, a revisão sistemática com foco na relação entre a proporção de crianças por adulto e o desenvolvimento das crianças na educação infantil realizada por Perlman et al. (2017) analisou 29 estudos nos quais essa razão variava de 5 a 14,5, com média de 8,65 crianças por adulto. A realidade 
brasileira é distinta, e nossas amostras sugerem que, na média, as turmas apresentam tamanhos maiores (entre 22 e 23 crianças), havendo considerável variação quando comparamos turmas dentro das mesmas faixas etárias e redes de ensino. Por exemplo, em uma das amostras do estudo longitudinal observamos turmas com 15 crianças e outras com 28; na outra amostra as diferenças eram ainda maiores de 6 crianças até 30 .

As análises com modelos de valor agregado para ambas as redes de ensino sugerem que o tamanho da turma explica parte da variação no aprendizado em linguagem. Não encontramos os mesmos resultados para o aprendizado em matemática. Os resultados obtiveram effect sizes que variaram de $-0,16$ a $-0,23$, sugerindo que quanto maior a turma, menor o aprendizado em linguagem. Uma forma de observar o tamanho do ganho é transformar o resultado em meses de aprendizado. ${ }^{14}$ Nesse caso, compa236 rando estudantes alocados em uma turma com 16 crianças e em uma turma com 28 crianças (turmas com número de alunos 1 desvio-padrão abaixo e 1 desvio-padrão acima da média da amostra B), os alunos alocados nas turmas menores apresentaram, em média, ganhos adicionais de três meses. A replicação dos resultados em ambas as redes estudadas reforça a necessidade de debater a importância de turmas menores. É interessante notar que, por vezes, o debate público para a faixa etária pré-escolar enfoca mais a necessidade de tempo integral na escola e pouco o tamanho das turmas. O estudo longitudinal analisou essas duas

\footnotetext{
14 Aqui utilizamos a abordagem de Higgins et al. (2013), que indicam uma interpretação do effect size em meses de progresso escolar, considerando o effect size de um desvio-padrão como equivalente a um ano de instrução. Os autores alertam que essa é uma equivalência aproximada e pode variar de acordo com a etapa da trajetória escolar em foco - tendendo a ser maior no início da escolarização e menor nos níveis subsequentes. Ainda assim, essa aproximação permite uma interpretação com mais sentido no que diz respeito à relevância pedagógica dos resultados obtidos, se comparados com interpretações restritas à significância estatística dos parâmetros estimados.
} 
dimensões (tempo diário na escola e tamanho da turma) e encontrou efeitos consistentes no aprendizado apenas associados ao tamanho da turma. Não se trata aqui de desconsiderar a importância do turno integral, mas sim de qualificar o debate, em especial quando o foco é a melhoria do aprendizado.

Ainda sobre o tamanho da turma, realizamos análises complementares para identificar se este exerce efeito moderador. Novamente, encontramos resultados consistentes para a medida da linguagem, sugerindo que alunos vulneráveis se beneficiam mais quando alocados em turmas menores, com um effect size de 0,14. Ou seja, na média, alunos vulneráveis apresentaram desenvolvimento ainda mais acelerado quando alocados em turmas menores. Caracterizamos como vulneráveis crianças que apresentam o ponto de partida - a medida inicial da linguagem - com 1 desvio-padrão abaixo da média da amostra e têm pais com baixa escolaridade. Estamos falando de um efeito potencial do tamanho da turma na diminuição das desigualdades educacionais. Estudos internacionais sugerem que aspectos relacionados à qualidade da estrutura das escolas - por exemplo, o tamanho da turma - explicam parte da variação na qualidade da interação entre professor e aluno (Cadima, 2017). Se isso é verdade, alunos vulneráveis têm suas oportunidades de aprender ampliadas, o que explicaria o ganho adicional observado no estudo longitudinal.

A terceira e última variável que analisamos neste artigo diz respeito aos programas escolares voltados especificamente para a educação infantil. Muitas redes de ensino diversificam a oferta da etapa pré-escolar, em parte oferecida em escolas dedicadas somente à educação infantil, e em parte em escolas que oferecem etapas da educação infantil integradas com séries do ensino fundamental. Programas voltados para escolas que oferecem somente educação infantil apresentam características específicas 
relacionadas a infraestrutura, materiais pedagógicos, formação de professores e gestão escolar focadas nas crianças de 0 a 5 anos. $O$ estudo longitudinal considerou essa variação da oferta de pré-escola dentro de cada rede para investigar se há ganhos adicionais no aprendizado, ou mesmo na diminuição das desigualdades educacionais, associados à frequência às escolas que oferecem somente educação infantil e participam de programas educacionais específicos para a primeira infância.

Em ambas as redes analisadas encontramos programas dedicados à educação infantil que apresentavam duas ou mais características supracitadas. Observamos que, em uma das redes, as escolas dedicadas à educação infantil tiveram um impacto consistente no aprendizado das crianças em matemática e linguagem. Os ganhos estimados em meses de aprendizado variavam de cinco, para linguagem, a três meses, para matemática. Esses são resultados muito promis238 sores, evidenciando que escolas com insumos e oferta especializada na educação infantil podem impulsionar o desenvolvimento das crianças.

Além disso, observamos novamente um efeito moderador associado à alocação das crianças nesse tipo de pré-escola. O desenvolvimento em linguagem das crianças vulneráveis foi mais acelerado em comparação com seus pares não vulneráveis, ou seja, crianças com um ponto de partida mais baixo tiveram um aprendizado de linguagem mais acelerado. As evidências encontradas sugerem o potencial desse programa para diminuir desigualdades educacionais caso seja ampliado para a totalidade das crianças matriculadas na rede. Análises complementares são necessárias para compreender os mecanismos a partir dos quais esse programa opera, de modo que as evidências encontradas possam subsidiar tomadas de decisão para a ampliação desse tipo de oferta na própria rede ou para a replicação do programa em outros municípios brasileiros. 


\section{Conclusão}

Este artigo apresentou os resultados de um estudo longitudinal realizado com crianças que ingressaram na pré-escola. Os dados com características longitudinais permitem estimar de forma adequada as características escolares e extraescolares associadas ao aprendizado das crianças. A medida do desenvolvimento das crianças, feita ao ingressarem no primeiro ano da pré-escola, serve como um parâmetro inicial que ajuda a descrever padrões de desigualdades educacionais no início da escolarização obrigatória e como estes se modificam ao longo dos dois primeiros anos na escola.

É importante que mais estudos longitudinais sejam realizados no Brasil para preencher uma importante lacuna no debate sobre eficácia escolar. Uma rápida análise dos estudos sobre o tema produzidos no Brasil demonstra que a maioria deles adota modelos transversais, que são inadequados para identificar fatores escolares associados com o aprendizado. A falta de estudos com desenhos robustos dificulta a identificação de programas capazes de combater a desigualdade educacional e melhorar a qualidade e a equidade dos sistemas públicos de ensino.

A temática da desigualdade irá provavelmente ganhar espaço com a grave crise sanitária de covid-19 vivida por diversos países do mundo, que tem como um de seus efeitos o fechamento das escolas por muitos meses. A ausência da escola e as condições de confinamento das famílias podem ter um efeito amplificador nas desigualdades educacionais, e isso irá demandar um grande esforço de gestores públicos, diretores e professores nos próximos meses e anos para reverter suas consequências. É premente a necessidade de estudos com desenhos robustos que permitam identificar com alto grau de certeza a eficácia de programas e estratégias pedagógicas. A construção de boa evidência cientifica é apenas o primeiro passo para a melhoria das políticas educacionais. Há ainda a necessidade da implementação 
competente e da avaliação permanente das políticas educacionais para aumentar a probabilidade de que todas as crianças aprendam o esperado nas diferentes etapas do processo de escolarização.

\section{Mariane Campelo Koslinski}

Doutora em Sociologia e professora da Faculdade de Educação da Universidade Federal do Rio de Janeiro (UFRJ). Coordenadora do Laboratório de Pesquisa em Oportunidades Educacionais (LaPOpE/UFRJ) e do GT 13 Educação e Sociedade, da Sociedade Brasileira de Sociologia. Desenvolve pesquisas sobre desigualdades de oportunidades educacionais e avaliação de políticas educacionais.

\section{Tiago Lisboa Bartholo}

Doutor em Educação pela Universidade Federal do Rio de Janeiro (UFRJ) e professor do Programa de Pós-Graduação em Educação da mesma instituição. Pesquisador do Laboratório de Pesquisa em Oportunidades Educacionais (LaPOpE/UFRJ). Tem interesse nos temas desigualdades educacionais e avaliação de impacto de políticas educacionais.

\section{Bibliografia}

AGUIAR, Daniel Kreuger de. 2018. A influência da aptidão física não aeróbica no desempenho cognitivo em alunos da pré-escola na rede municipal do Rio de Janeiro. Dissertação de Mestrado em Educação. Rio de Janeiro: Universidade Federal do Rio de Janeiro.

AGUIAR, Daniel Kreuger de; BARTHOLO, Tiago Lisboa. 2019.

A associação entre a aptidão física não-aeróbica e o desempenho cognitivo de crianças na pré-escola. Ciência Ė Desenvolvimento, v. 12, n. 3, pp. 725-740.

AGUIAR, Daniel Kreuger de; BARTHOLO, Tiago Lisboa; TAVARES JÚNIOR, Fernando. 2019. Relações entre a aptidão física não-aeróbica e o desempenho matemático em crianças. Lecturas: Educación Física y Deportes, v. 24, n. 251, pp. 15-25. 
ALVES, Maria Teresa Gonzaga; XAVIER, Flavia Pereira. 2016. Construção de indicadores para descrever desigualdades de aprendizado na Prova Brasil. Estudos em Avaliação Educacional, v. 27, n. 66, pp. 782-815.

ALVES, Maria Teresa Gonzaga; SOARES, José Francisco; XAVIER, Flavia Pereira. 2016. Desigualdades Educacionais no ensino fundamental de 2005 a 2013: hiato entre grupos sociais. Revista Brasileira de Sociologia, v. 4, n. 7, pp. 49-81.

BARNETT, W. Steven; BOOCOCK, Sarane Spence (ed.). 1998. Early care and education for children in poverty: promises, programs, and long-term results. Albany: State University of New York Press.

BARTHOLO, Tiago Lisboa; COSTA, Marcio. 2016. Evidence of a school composition effect in Rio de Janeiro public schools. Ensaio: Avaliação e Políticas Públicas em Educação, v. 24, n. 92, pp. 498-521.

BARTHOLO, Tiago Lisboa; KOSLINSKI, Mariane Campelo. 2020. First two years at school: evidence-based policy for early childhood education in Brazil. In: GORARD, Stephen (org.). Getting evidence into education: evaluating the routes to policy and practice. Abingdon: Routledge. pp. 110-118.

BARTHOLO, Tiago Lisboa et al. 2020a. The use of cognitive instruments for research in early childhood education: constraints and possibilities in the Brazilian context. Pro-Posições, v. 31, e20180036.

BARTHOLO, Tiago Lisboa et al. 2020b. What do children know upon entry to pre-school in Rio de Janeiro? Ensaio: Avaliação e Políticas Públicas em Educação, v. 28, n. 107, pp. 292-313.

BONAMINO, Alicia et al. 2010. Os efeitos das diferentes formas de capital no desempenho escolar: um estudo à luz de Bourdieu e de Coleman. Revista Brasileira de Educação, v. 15, n. 45, pp. 487-499.

BOONE, William J. 2016. Rasch analysis for instrument development: why, when, and how? CBE: Life Sciences Education, v. 15, n. 4, pp. 1-7.

BRASIL. 2014. Lei no 13.005, de 25 de junho 2014. Aprova o Plano Nacional de Educação - PNE e dá outras providências. Diário Oficial da União: seção 1, p. 1, 26 jun. 2014. Edição extra.

BROOKE, Nigel; BONAMINO, Alicia (org.). 2011. Geres 2005: razões e resultados de uma pesquisa longitudinal sobre a eficácia escolar. Rio de Janeiro: Walprint.

CADIMA, Joana. 2017. QualityMatters: how and under what conditions does quality in early education and care matter? A study across four European countries. Nauki o Wychowaniu: Studia Interdyscyplinarne, v. 2, n. 5, pp. 190-199. 
CAMPBELL, Frances A. et al. 2001. The development of cognitive and academic abilities: growth curves from an early childhood educational experiment. Developmental Psychology, v. 37, n. 2, pp. 231-242.

CAMPOS, Maria Malta et al. 2011a. A contribuição da educação infantil de qualidade e seus impactos no início do ensino fundamental. Educação $e$ Pesquisa, v. 37, n. 1, pp. 15-33.

CAMPOS, Maria Malta et al. 2011b. A qualidade da educação infantil: um estudo em seis capitais brasileiras. Cadernos de Pesquisa, v. 41, n. 142, pp. 20-54.

CASTRO, Carla Maria Motta do Valle. 2017. Avaliação do aprendizado no ciclo de alfabetização de alunos da rede municipal do Rio de Janeiro. Dissertação de Mestrado em População, Território e Estatísticas Públicas. Rio de Janeiro: Escola Nacional de Ciências Estatísticas.

COLEMAN, James S. et al. 1966. Equality of educational opportunity. Washington, DC: US Government Printing Office.

CRAHAY, Marcel; BAYE, Ariane. 2013. Existem escolas justas e eficazes? Esboço de resposta baseado no Pisa 2009. Cadernos de Pesquisa, v. 43, n. 150 , pp. $858-883$.

DAMIANI, Magda Floriana et al. 2011. Educação infantil e longevidade escolar: dados de um estudo longitudinal. Estudos de Avaliação Educacional, v. 22, n. 50, pp. 515-532.

FINN, Jeremy D.; ACHILLES, Charles M. 1999. Tennessee's class size study: findings, implications, misconceptions. Educational Evaluation and Policy Analysis, v. 21, n. 2, pp. 97-109.

FRANCO, Creso; BROOKE, Nigel; ALVES, Fátima. 2008. Estudo longitudinal sobre qualidade e equidade no ensino fundamental brasileiro: Geres 2005. Ensaio: Avaliação e Políticas Públicas em Educação, v. 16, n. 61, pp. 625-638.

FRANCO, Creso et al. 2007. Qualidade e equidade em educação: reconsiderando o significado de "fatores intra-escolares". Ensaio: Avaliação e Políticas Públicas em Educação, v. 15, n. 55, pp. 277-298.

FITZ-GIBBON, Carol. 1996. Monitoring education: indicators, quality and effectiveness. London: Continuum.

GOLDSTEIN, Harvey. 1997. Methods in school effectiveness research. School Effectiveness and School Improvement, v. 8, n. 4, pp. 369-395.

HARMS, Thelma; CLIFFORD, Richard M.; CRYER, Debby. 2005. Early Childhood Environment Rating Scale: revised edition. New York: Teachers College Press.

HASENBALG, Carlos; SILVA, Nelson do Valle. 2003. Origens e destinos. Rio de Janeiro: Topbooks. 
HIGGINS, Steve et al. 2013. The Sutton Trustt-Education Endowment Foundation teaching and learning toolkit: technical appendices. London: Education Endowment Foundation. Disponível em: https:/ /bit.ly/2XPAqT6. Acesso em: 12 out. 2018.

HOWES, Carollee et al. 2008. Ready to learn? Children's pre-academic achievement in pre-kindergarten programs. Early Childhood Research Quarterly, v. 23, n. 1, pp. 27-50.

KOSLINSKI, Mariane Campelo; BARTHOLO, Tiago Lisboa. 2019. Impacto dos espaços de desenvolvimento infantil no primeiro ano na pré-escola. Estudos em Avaliação Educacional, v. 30, n. 73, pp. 280-311.

LEE, Valerie. 2004. Medidas educacionais: avaliando a eficácia das escolas em termos de excelência e de equidade. In: BONAMINO, Alicia, BESSA, Nícia; FRANCO, Creso (org.). Avaliação da educação básica. Rio de Janeiro: Editora PUC-Rio; São Paulo: Loyola. pp. 13-43.

MARINO, Leandro Lins. 2016. Análise de dados com característica longitudinal em educação: um estudo sobre o município do Rio de Janeiro. Dissertação de Mestrado em População, Território e Estatísticas Públicas.

Rio de Janeiro: Escola Nacional de Ciências Estatísticas.

MENDONÇA, Luiza de Souza Ferreira de. 2019. Conhecimento e interpretação de dados educacionais: o potencial das devolutivas e da capacitação para a compreensão e utilização de relatórios pedagógicos. Dissertação de Mestrado em Educação. Rio de Janeiro: Universidade Federal do Rio de Janeiro.

MERRELL, Christine; BAILEY, Katharine. 2008. Predicting achievement in the early years: how influential is personal, social and emotional development. Trabalho apresentado na International Association for Educational Assessment Conference, Cambridge, setembro de 2008. Disponível em: https://cutt.ly/dd6wXHN. Acesso em: 17 ago. 2020.

MONT'ALVÃO, Arnaldo. 2011. Estratificação educacional no Brasil do século XXI. Dados, v. 54, n. 2, pp. 389-430.

NICHD Early Child Care Research Network. 2006. Child-care effect sizes for the NICHD Study of Early Child Care and Youth Development. American Psychologist, v. 61, n. 2, pp. 99-116.

OLIVEIRA, André Luiz Regis de. 2020. "Um, dois, três": o desenvolvimento da contagem no início da escolarização obrigatória. Tese de Doutorado em Educação. Rio de Janeiro: Universidade Federal do Rio de Janeiro.

PEISNER-FEINBERG, Ellen S. et al. 2001. The relation of preschool child-care quality to children's cognitive and social developmental trajectories through second grade. Child Development, v. 72, n. 5, pp. 1534-1553. 
PERLMAN, Michal. et al. 2017. Child-staff ratios in early childhood education and care settings and child outcomes: a systematic review and meta-analysis. PLoS ONE, v. 12, n. 1, e0170256.

PIANTA, Robert C.; LA PARO, Karen M.; HAMRE, Bridget K. 2008. Classroom Assessment Scoring System: manual Pre-K. Baltimore: Paul H. Brookes Publishing.

RIBEIRO, Carlos Antônio Costa; CENEVIVA, Ricardo; ALVES DE BRITO, Murillo Marschner. 2015. Estratificação educacional entre jovens no Brasil. In: ARRETCHE, Marta (org.). Trajetórias das desigualdades: como o Brasil mudou nos últimos 50 anos. São Paulo: Editora Unesp. pp. 79-108. RODRIGUES, Blenda Luize Chor. 2018. A caixa preta ainda não foi aberta: uma revisão da literatura sobre a relação família-escola na educação infantil. Trabalho de Conclusão de Curso de Licenciatura em Pedagogia. Rio de Janeiro: Universidade Federal do Rio de Janeiro.

SAMMONS, Pam et al. 2006. Influences on children's attainment and progress in Key Stage 2: cognitive outcomes in Year 6: Effective Pre-School and Primary Education 3-11 Project (EPPE 3-11). London: Department for Children, Schools and Families. (Research Report DCSF-RR048).

SANTOS, Karina Porciuncula de Almeida Rodrigues. 2020. O primeiro ano na pré-escola: a relação entre desenvolvimento cognitivo, comportamento $e$ habilidades socioemocionais. Dissertação de Mestrado em Educação. Rio de Janeiro: Universidade Federal do Rio de Janeiro.

SCHWEINHART, Lawrence J.; WEIKART, David P. 1990. The High/ Scope Perry Preschool Study: implications for early childhood care and education. Prevention in Human Services, v. 7, n. 1, pp. 109-132.

SILVA, Camila Martins de Souza et al. 2019. Educação infantil em Boa Vista: medindo qualidade e resultados na educação infantil: 2019. São Paulo: Lepes: Fundação Maria Cecília Souto Vidigal. Disponível em: https://bit.ly/33ZiCbU. Acesso em: 30 mar. 2020.

SIQUEIRA, Carlos Eduardo Borges da Cruz. 2019. A relação entre a gestão democrática e a aprendizagem dos alunos na pré-escola na rede pública municipal do Rio de Janeiro. Dissertação de Mestrado em Educação. Rio de Janeiro: Universidade Federal do Rio de Janeiro.

SIRIN, Selcuk R. 2005. Socioeconomic status and academic achievement: a meta-analytic review of research. Review of Educational Research, v. 75, n. 3, pp. 417-453.

SOARES, José Francisco. 2004. Qualidade e equidade na educação básica brasileira: a evidência do Saeb-2001. Archivos Analíticos de Políticas Educativas, v. 12, n. 38, pp. 1-24. 
SOARES, José Francisco; DELGADO, Victor Maia Senna. 2016. Medida das desigualdades de aprendizado entre estudantes de ensino fundamental. Estudos em Avaliação Educacional, v. 27, n. 66, pp. 754-780.

SOARES, Tufi Machado. 2003. Influência do professor e do ambiente em sala de aula sobre a proficiência alcançada pelos alunos avaliados no Simave-2002. Estudos em Avaliação Educacional, n. 28, pp. 103-124.

SYLVA, Kathy; TAGGART, Brenda; SIRAJ-BLATCHFORD, Iram. 2003. Ecers-R: the four curricular subscales extension to the Early Childhood Environment Rating Scale (Ecers-R). Stoke on Trent, UK: Trentham Books. SYLVA, Kathy et al. 2006. Capturing quality in early childhood through environmental rating scales. Early Childhood Research Quarterly, v. 21, n. 1, pp. 76-92.

SYLVA, Kathy et al. 2010. Early childhood matters: evidence from the Effective Pre-school and Primary Education project. London: Routledge.

TAVARES JÚNIOR, Fernando (org.). 2018. Rendimento educacional no Brasil. Juiz de Fora: Obeduc.

TYMMS, Peter. 2004. Effect sizes in multilevel models. In: SCHAGEN, Ian; ELLIOT, Karen (ed.). But what does it mean? The use of effect sizes in educational research. Slough, UK: National Foundation for Educational Research. pp. 55-66.

TYMMS, Peter; MERRELL, Christine; HENDERSON, Brian. 1997. The first year at school: a quantitative investigation of the attainment and progress of pupils. Educational Research and Evaluation, v. 3, n. 2, pp. 101-118.

TYMMS, Peter; MERRELL, Christine; HENDERSON, Brian. 2000.

Baseline assessment and progress during the first three years at school. Educational Research and Evaluation, v. 6, n. 2, pp. 105-129.

TYMMS, Peter; MERRELL, Christine; JONES, Paul. 2004. Using baseline assessment data to make international comparisons. British Educational Research Journal, v. 30, n. 5, pp. 673-689.

TYMMS, Peter et al. 2009. The first seven years at school. Educational Assessment, Evaluation and Accountability, v. 21, n. 1, pp. 67-80.

WALSTON, Jill; WEST, Jerry. 2004. Full-day and half-day kindergarten in the United States: findings from the Early Childhood Longitudinal Study, Kindergarten Class of 1998-99. Washington, DC: National Center for Education Statistics. 


\title{
DESIGUALDADES DE OPORTUNIDADES EDUCACIONAIS NO INÍCIO DA TRAJETÓRIA ESCOLAR NO CONTEXTO BRASILEIRO
}

\author{
MARIANE CAMPELO KOSLINSKI
}

\section{TIAGO LISBOA BARTHOLO}

Resumo: Este artigo discute a importância de estudos com desenhos longitudinais sobre o início da escolarização obrigatória para compreender padrões de desigualdades de oportunidades educacionais e as potencialidades e fragilidades dos desenhos dos estudos de eficácia escolar produzidos no Brasil. Descreve-se um estudo longitudinal desenvolvido em dois municípios brasileiros, com amostras que incluíram 123 escolas (6.379 crianças) e algumas evidências que permitem compreender tendências e nuances relacionadas às desigualdades educacionais no início da escolarização obrigatória no contexto brasileiro. Este texto também discute como o desenho do estudo possibilita identificar programas educacionais e características escolares capazes de atenuar a distância da aprendizagem de crianças de origens socioeconômicas distintas.

Palavras-chave: Desigualdades de Oportunidades Educacionais; Estudos Longitudinais; Pré-Escola; Eficácia Escolar; Equidade.

\section{INEQUALITIES IN EDUGATIONAL OPPORTUNITIES AT THE BEGINNING OF THE EDUCATIONAL TRAJECTORY IN BRAZIL}

Abstract: This paper discusses the importance of studies with longitudinal design at the beginning of compulsory schooling to understand patterns of inequality in educational opportunities and the strengths and weaknesses of the research design of school effectiveness studies, focusing on the beginning of the educational trajectory in Brazil. It describes a longitudinal study carried out in two Brazilian cities in samples that included 123 schools (6373 children) 
and some evidence that clarifies certain trends and nuances of educational inequalities at the beginning of compulsory schooling in Brazil. We also discuss how study design permits a more accurate identification of educational programs and school characteristics that are able to bridge the learning gap between children of different socioeconomic backgrounds.

Keywords: Inequalities of Educational Opportunities; Longitudinal Studies; Preschool; School Effectiveness; Equity.

Recebido: 03/06/2020Ａprovado: 05/08/2020 